Volume 23 (2018) 42-46

DOI: $10.24330 /$ ieja.373640

\title{
A NOTE ON FINITE GROUPS WITH FEW TI-SUBGROUPS
}

\author{
Jiangtao Shi, Jingjing Huang and Cui Zhang \\ Received: 10 June 2016; Revised: 29 July 2017; Accepted: 10 August 2017 \\ Communicated by Abdullah Harmancı \\ Abstract. In [Comm. Algebra, 43(2015), 2680-2689], finite groups all of \\ whose metacyclic subgroups are TI-subgroups have been classified by $\mathrm{S}$. Li, \\ Z. Shen and N. Du. In this note we investigate a finite group all of whose \\ non-metacyclic subgroups are TI-subgroups. We prove that $G$ is a group all \\ of whose non-metacyclic subgroups are TI-subgroups if and only if all non- \\ metacyclic subgroups of $G$ are normal. Furthermore, we show that a group all \\ of whose non-cyclic subgroups are TI-subgroups has a Sylow tower.
}

Mathematics Subject Classification (2010): 20D10

Keywords: Non-metacyclic subgroup, TI-subgroup, normal, solvable, Sylow tower

\section{Introduction}

In this paper all groups are assumed to be finite. Let $G$ be a group. If $G$ has a cyclic normal subgroup $H$ such that $G / H$ is cyclic, then $G$ is said to be a metacyclic group. It is obvious that a cyclic group is also a metacyclic group but a metacyclic group might not be a cyclic group.

Let $G$ be a group and $K$ a subgroup of $G$. If $K^{g} \cap K=1$ or $K$ for all $g \in G$, then $K$ is called a TI-subgroup of $G$. It is clear that every normal subgroup of a group $G$ is a TI-subgroup of $G$ but a TI-subgroup of $G$ might not be a normal subgroup of $G$.

In [2], Mousavi, Rastgoo and Zenkov characterized non-nilpotent groups all of whose cyclic subgroups are TI-subgroups. As a generalization, the first author and the third author [4] proved that $G$ is a group all of whose non-cyclic subgroups are TI-subgroups if and only if all non-cyclic subgroups of $G$ are normal. Recently, $\mathrm{Li}$, Shen and Du [1] classified groups all of whose metacyclic subgroups are TIsubgroups.

J. Shi was supported in part by Shandong Provincial Natural Science Foundation, China (ZR2017MA022) and NSFC (11561021 and 11761079). C. Zhang was supported in part by NSFC (11201403 and 11561021). 
Motivated by these results, the main goal of this note is to investigate the structure of a group all of whose non-metacyclic subgroups are TI-subgroups. For such groups, we obtain the following result whose proof is given in Section 2.

Theorem 1.1. A group $G$ is a group all of whose non-metacyclic subgroups are TI-subgroups if and only if all non-metacyclic subgroups of $G$ are normal.

Furthermore, we have that a group all of whose non-metacyclic subgroups are TI-subgroups is solvable, the proof of which is given in Section 3.

Theorem 1.2. Let $G$ be a group all of whose non-metacyclic subgroups are TIsubgroups, then $G$ is solvable.

Remark 1.3. If we assume that $G$ is a group all of whose non-metaabelian subgroups are TI-subgroups, then $G$ might not be solvable. Observing that the alternating group $A_{5}$ is a non-solvable example.

Remark 1.4. Note that the symmetric group $S_{4}$ has only two non-metacyclic subgroups $A_{4}$ and $S_{4}$, then $S_{4}$ is a group with all non-metacyclic subgroups being TI-subgroups. This example shows that the group in Theorem 1.2 might not be supersolvable.

Remark 1.5. The example $S_{4}$ also shows that the group in Theorem 1.2 might not have a Sylow tower.

In Remark 1 of [4], the first author and the third author indicated that a group all of whose non-cyclic subgroups are TI-subgroups is solvable but it might not be supersolvable. In this note, we prove that a group all of whose non-cyclic subgroups are TI-subgroups has the following property:

Theorem 1.6. Suppose that $G$ is a group all of whose non-cyclic subgroups are TI-subgroups, then $G$ has a Sylow tower.

The proof of Theorem 1.6 is given in Section 4. However, the alternating group $A_{4}$ shows that the group in Theorem 1.6 might not have a Sylow tower of supersolvable type.

\section{Proof of Theorem 1.1}

Proof. Since the sufficiency part is clearly holds, we only need to prove the necessity part.

Suppose that the necessity part is not true. Assume that $H$ is the largest nonmetacyclic subgroup of $G$ which is not normal in $G$. Let $M$ be a subgroup of $G$ 
such that $H$ is maximal in $M$. It is clear that $M$ is non-metacyclic. By the choice of $H$, we have that $M$ is normal in $G$.

Case I: Assume that $H$ is normal in $M$.

Take $g \in G \backslash N_{G}(H)$, one has that $H^{g}$ is normal in $M^{g}=M$. Moreover, one has $H \cap H^{g}=1$ since $H$ is a TI-subgroup of $G$. Then $M=H \times H^{g}$. It follows that $H$ is isomorphic to a cyclic group of prime order, this contradicts that $H$ is non-metacyclic.

Case II: Assume that $H$ is not normal in $M$.

One has $H=N_{M}(H)$. Since $H$ is also a TI-subgroup of $M$, it follows that $M$ is a Frobenius group with $H$ being its Frobenius complement. Let $N$ be the Frobenius kernel of $M$. Then $M=N \rtimes H$.

Note that $H$ is maximal in $M$. One has that $N$ is a minimal normal subgroup of $M$. Since $N$ is nilpotent by [3, Theorem 10.5.6 (i)], we have that $N$ is an elementary abelian group of prime-power order.

Assume $N=Z_{p} \times Z_{p} \times \cdots \times Z_{p}=Z_{p}{ }^{m}$, where $p$ is a prime and $m \geq 1$ is a positive integer.

(1) If $m=1$, then $N=Z_{p}$ is a cyclic group of order $p$. By N/C-theorem [3, Theorem 1.6.13], we have $H \cong M / N=N_{M}(N) / C_{M}(N) \lesssim \operatorname{Aut}(\mathrm{N})=\mathrm{Z}_{\mathrm{p}-1}$. It follows that $H$ is a cyclic group, a contradiction.

(2) If $m>1$, one has that $N$ is non-cyclic. For every subgroup $R \neq 1$ of $H$, it is clear that $N \rtimes R$ is also a Frobenius group. We will show that $N \rtimes R$ is non-metacyclic.

Otherwise, assume that $N \rtimes R$ is metacyclic. Then there exists a cyclic normal subgroup $E$ of $N \rtimes R$ such that $(N \rtimes R) / E$ is cyclic. Since $N \rtimes R$ is a Frobenius group, one has either $E<N$ or $N \leq E$. It is obvious that $N \leq E$ is impossible since $N$ is non-cyclic. If $E<N$, then $(N \rtimes R) / E=(N / E) \rtimes(R E / E)$ is still a Frobenius group, this contradicts that $(N \rtimes R) / E$ is cyclic. Thus $N \rtimes R$ is non-metacyclic.

By the hypothesis, one has that $N \rtimes R$ is a TI-subgroup of $M$. Note that for every $x \in M$ we always have $(N \rtimes R)^{x} \cap(N \rtimes R)=\left(N^{x} \rtimes R^{x}\right) \cap(N \rtimes R)=$ $\left(N \rtimes R^{x}\right) \cap(N \rtimes R) \geq N \neq 1$. It follows that $N \rtimes R$ is normal in $M$. Then $R=(N \cap H) R=N R \cap H$ is normal in $H$. By the arbitrary choice of $R$, we have that $H$ is a Dedekind-group.

By [3, Theorem 5.3.7], one has that $H$ is either abelian or the direct product of a quaternion group of order 8 , an elementary abelian 2-group and an abelian group of odd order. Note that the Sylow $p$-subgroups of $H$ are cyclic if $p>2$ and cyclic or generalized quaternion if $p=2$ by [3, Theorem 10.5.6 (ii)]. 
If $H$ is abelian, then $H$ is cyclic, a contradiction.

If $H$ is a non-abelian Dedekind group, then the Sylow 2-subgroup of $H$ is the direct product of a quaternion group of order 8 and an elementary abelian 2-group, which is also a generalized quaternion group. Since the center of a generalized quaternion 2-group has order 2, we must have that the Sylow 2-subgroup of $H$ is a quaternion group $Q_{8}$ of order 8. It follows that $H=Q_{8} \times Z_{t}$, where $t$ is an odd number. It is easy to see that $H$ is a metacyclic group, a contradiction.

By all above arguments, we know that there is no non-normal non-metacyclic subgroups in $G$. Hence all non-metacyclic subgroups of $G$ are normal in $G$.

\section{Proof of Theorem 1.2}

Proof. By Theorem 1.1, we only need to prove that if all non-metacyclic subgroups of $G$ are normal then $G$ is solvable.

Suppose the theorem is not true. Let $G$ be a counterexample of the smallest order. Then $G$ is a minimal non-solvable group, which implies that $G / \Phi(G)$ is a minimal non-abelian simple group, where $\Phi(G)$ is the Frattini subgroup of $G$.

It is obvious that $G$ has no normal maximal subgroups. By the hypothesis, one has that all maximal subgroups of $G$ are metacyclic. It follows that all maximal subgroups of $G$ are supersolvable. By [3, Theorem 10.3.4], one has that $G$ is solvable, a contradiction.

Hence the assumption is not true and then $G$ is solvable.

\section{Proof of Theorem 1.6}

Proof. By [4, Theorem 1], we only prove that if all non-cyclic subgroups of $G$ are normal then $G$ has a Sylow tower.

Let $G$ be a counterexample of minimal order. Assume that $p$ is the smallest prime divisor of $|G|$ and $P$ is a Sylow $p$-subgroup of $G$.

We divide the proof into two cases depending on whether $P$ is cyclic or not.

Case I: Suppose that $P$ is cyclic. By a corollary of Burnside's theorem [3, Theorem 10.1.9], we have that $G$ is $p$-nilpotent, that is, $G$ has a normal $p$-complement. Let $H$ be a normal $p$-complement of $P$ in $G$. Then $G=H \rtimes P$, the semidirect product of $H$ and $P$. Since $H$ is also a group all of whose non-cyclic subgroups are normal and $|H|<|G|$, by the minimality of $G$, we have that $H$ has a Sylow tower: $H \triangleright H_{1} \triangleright H_{2} \triangleright \cdots \triangleright H_{s-1} \triangleright H_{s}=1$. Note that for every $1 \leq i \leq s-1$ one has that $H_{i}$ is a Hall-subgroup of $H$, then $H_{i}$ is a characteristic subgroup of $H$. Since $H$ is normal in $G$, one has that $H_{i}$ is normal in $G$ for every $1 \leq i \leq s-1$. It follows 
that $G$ has a Sylow tower: $G \triangleright H \triangleright H_{1} \triangleright H_{2} \triangleright \cdots \triangleright H_{s-1} \triangleright H_{s}=1$. This contradicts that we assume that $G$ has no Sylow tower.

Case II: Suppose that $P$ is non-cyclic. By the hypothesis, one has that $P$ is normal in $G$. Considering the quotient group $G / P$. Since $G / P$ is also a group all of whose non-cyclic subgroups are normal and $|G / P|<|G|$, by the minimality of $G$, one has that $G / P$ has a Sylow tower: $G / P \triangleright G_{1} / P \triangleright \cdots \triangleright G_{t-1} / P \triangleright G_{t} / P=1$. It is clear that $G \triangleright G_{1} \triangleright \cdots \triangleright G_{t-1} \triangleright G_{t}=P \triangleright 1$ is a Sylow tower of $G$. It is also a contradiction.

Case I and Case II imply that the counterexample of minimal order does not exist. Hence $G$ has a Sylow tower.

Acknowledgment. The authors are thankful to the referee who gives valuable comments.

\section{References}

[1] S. Li, Z. Shen and N. Du, Finite groups with few TI-subgroups, Comm. Algebra, 43(7) (2015), 2680-2689.

[2] H. Mousavi, T. Rastgoo and V. Zenkov, The structure of non-nilpotent CTIgroups, J. Group Theory, 16(2) (2013), 249-261.

[3] D. J. S. Robinson, A Course in the Theory of Groups, Second Edition, Graduate Texts in Mathematics, 80, Springer-Verlag, New York, 1996.

[4] J. Shi and C. Zhang, Finite groups in which some particular subgroups are TI-subgroups, Miskolc Math. Notes, 14(3) (2013), 1037-1040.

Jiangtao Shi (Corresponding Author) and Cui Zhang

School of Mathematics and Information Science

Yantai University

264005 Yantai, China

e-mails: jiangtaoshi@126.com (J. Shi)

cuizhang2008@gmail.com (C. Zhang)

\section{Jingjing Huang}

Faculty of Science and Technology

Communication University of China

100024 Beijing, China

e-mail: 1543460315@qq.com 